\title{
Pré e Pós operatório nas DII \\ Otimização \\ pré-operatória e \\ manejo pró-operatório
}

\section{Cristina Flores}

\section{Introdução}

Apesar do avanço na terapia das doenças inflamatórias intestinais (DII), aproximadamente 50\% dos pacientes com Doença de Crohn necessitam cirurgia nos primeiros 10 anos de doença, e 15\% dos pacientes com Retocolite ulcerativa (RCU) necessitam colectomia ao longo da vida ${ }^{1,2,3}$. Os pacientes com DIl possuem um potencial para múltiplas intervenções cirúrgicas e a possibilidade de desenvolvimento da síndrome do intestino curto sempre causa preocupação adicional. Assim, muitas vezes os pacientes chegam à cirurgia desnutridos, em uso de imunossupressores, cronicamente subocluídos ou com sepse abdominal. Todos estes são fatores que aumentam as complicações pós-operatórias. Consequentemente, a otimização pré-operatória é de suma importância para reduzir a morbidade das intervenções cirúrgicas. O manejo pós-operatório deve incluir a prevenção e a vigilância de condições que podem levar a morte com a sepse e o tromboembolismo.

\section{Nutrição}

A desnutrição pré-operatória aumenta as taxas de complicações pós-operatórias, incluindo o maior risco de infecções. A desnutrição e a perda de peso significativa (> 10\% do peso), pode variar de $20-85 \%$ dependendo dos parâmetros utilizados para definição do estado nutricional ${ }^{4,5}$. A desnutrição aumenta a mortalidade intrahospitalar (Razão de chance 3.49, IC (95\%) 2.89-4.23) e tempo de internação em 2 vezes. (6) Alguns autores demonstraram que a hipoalbuminemia (albumina 
$<3,5 \mathrm{~g} / \mathrm{dl}$ ) é um fator de risco para deiscência da anastomose ${ }^{7,5}$.

Todos os pacientes devem ser avaliados do ponto de vista de desnutrição proteico-calórica, além de deficiências específicas de vitaminas, micronutrientes e ferro. Devido a frequência de deficiência de vitamina B12, ferro, zinco e vitamina D merecem uma atenção especial. Dependendo da intensidade da diarreia, pode haver níveis séricos baixos de potássio, magnésio, cálcio e fosfato.

Os suplementos nutricionais por via oral costumam ser bem tolerados. A Sociedade Europeia para nutrição clínica e metabolismo recomenda que pelo menos $600 \mathrm{kcal} /$ day possam ser ofertadas aos pacientes. No entanto, se as necessidades nutricionais calculadas não podem ser atingidas pela alimentação oral e suplementos deve-se utilizar a nutrição enteral. Não há diferença nos resultados nutricionais entre as fórmulas poliméricas, monoméricas ou elementares. Além da eficácia na correção do estado nutricional, a nutrição enteral é capaz de induzir melhora do processo inflamatório intestinal e, consequentemente, reduzir a necessidade de corticoide. Não existem evidências de que a nutrição parenteral total possua qualquer benefício sobre a dieta enteral e só deve ser utilizada nas situações de impossibilidade do uso do tubo digestivo como nas fístulas de alto débito, obstrução intestinal ou síndrome do intestino curto. Neste contexto, a nutrição parenteral se justifica e é eficaz. Não esquecendo que o índice de complicações associadas a sepse, a trombose pelo cateter central e a insuficiência hepática não são desprezíveis ${ }^{5,8,9}$.

Anemia é a manifestação extra-intestinal mais frequente na DII, variando entre 36 e $88 \%$. Ela ocorre por vários mecanismos como a anemia de doença crônica, deficiência de vitamina B 12 e, frequentemente, por deficiência de ferro. A anemia por deficiência de ferro possue também um impacto sobre o desempenho do sistema imunológico e risco de infecções. Existe também, uma relação entre déficit de ferro, trombocitose e tromboembolismo ${ }^{9,10}$. A reposição de ferro por via parenteral deve ser considerada em todo paciente com $\mathrm{Hb}<10 \mathrm{~g} / \mathrm{dL}$, com $\mathrm{Hb}>10 \mathrm{~g} / \mathrm{dl}$ que não tolere a reposição de ferro via oral ou com doença ativa ${ }^{10}$.

\section{Sepse}

A sepse abdominal, devido a fítulas ou abscessos, é uma complicação comum na Doença de Crohn, sendo um fator de risco inde- 
pendente para complicações pós-operatórias. Podendo ocorrer em até $30 \%$ dos $\operatorname{casos}^{11,12}$. Sempre que possível os abscessos devem ser tratados com drenagem percutânea e antibióticos na tentativa de evitar a cirurgia em um cenário de urgência. Esta conduta pode diminuir o índice de complicações pós-operatórias, evitando a necessidade de construção de um ostoma e permitindo a melhora das condições gerais do paciente antes da cirurgia. Em algumas situações a drenagem percutânea pode proporcionar a resolução do abscesso evitando a necessidade de intervenção cirúrgica ${ }^{13,14}$.

\section{Medicação Imunossupressoras e Biológicos no Período Pré-Operatório}

O uso de corticoide dose quivalente a prednisona $20 \mathrm{mg}$ por dia por mais do que 6 semanas é um fator de risco para complicações pós- cirúrgicas. Assim, os corticoides devem ser retirados, se possível, antes da cirurgia. Nguyen e colaboradores recentemente avaliaram a repercussão do uso de corticoides no período pré-operatório. Encontraram que o uso pré-operatório de esteroides foi associado com maior risco de complicações infecciosas no pós-opreatório (DC 22.6\% x 18.5\%, e RCU $30.1 \%$ x. 22.5\%, p<0.0001). Neste estudo também foi observado que a presença de anemia e perda de peso no período pré-operatório aumentaram o risco de complicações ${ }^{15}$. Para os pacientes que estão usando cornicamente esteroides e tem uma cirurgia eletiva a frente, deve-se considerar a suspensão dos cortioidees pelo menos 30 dias antes do procedimento sempre que possível. Os pacientes recebendo doses terapêuticas de corticoide que forem submetidos a procedimento cirúrgico não necessitam rotineiramente de doses suplementares de estresse para evitar a insuficiência adrenal, desde que continuem a receber a dose diária habitual a que vinham sendo submetidos, e sejam monitorados para sinais de insuficiência adrenal ${ }^{16}$.

$\mathrm{O}$ uso de imumomoduladores, incluindo a ciclosporina e as tiopurinas, não tem sido associado com risco aumentado de complicações no pos-op ${ }^{17}$.

Existe uma grande controvérsia sobre o aumento da taxa de complicações pos-op de cirurgias abdominais com o uso de anti-TNF. O intervalo de segurança permanece por ser determinado. O TNF $\alpha$ é um fator chave na resposta imunológica. Sendo assim, a inibição do TNF pela 
terapia anti-TNF poderia produzir um efeito deletério levando a um maior índice de complicações pós-operatórias. Existem dados conflitantes na literatura. Não há consenso entre os especialistas. Um estudo mais recente realizado por Syed e colaboradores avaliaram 325 cirurgias abdominais realizadas em pacientes com DC, destes 150 procedimentos foram realizados em pacientes com uso prévio de anti-TNFs comparados com outros 175 sem exposição prévia. Foi encontrada uma maior taxa de complicações infecciosas no grupo que utilizou anti-TNF em relação ao grupo controle (36 \% x $25 \%$, p= 0.05). Em análise multivariada demonstraram uma razão de chance de 2.43 (IC - 1.18 - 5.03) ${ }^{18}$. Uma metanálise recente também demonstra um pequeno aumento no risco de complicações infecciosas nos pacientes com DC, porém não ocorrendo nos pacientes com RCU ${ }^{19}$. Outro estudo também recentemente publicado não demonstra aumento da taxa de complicações ${ }^{20}$. Assim, embora a maioria dos estudos aponte para um aumento de risco, devemos aguardar dados coletados de forma prospectiva para termos conclusões mais definitivas sobre este tópico. A farmacocinética dos anticorpos monoclonais demonstra que as concentrações terapêuticas geralmente persistem por pelo menos 8 semanas após a infusão. Quando o paciente tiver que realizar uma cirurgia programada, pode-se planejar a intervenção cirúrgica para o final destas 8 semanas, ou seja, realizando a cirurgia em um momento em que os níveis séricos estejam mais baixos.

\section{Referências bibliográficas}

1. Solberg IC, Vatn MH, Hoie O et al. Clinical course in Crohn's disease: results of a Norwegian population-based ten-year follow-up study. Clin Gastroenterol Hepatol 2007; 5: 1430 - 8.

2. Nguyen GC, Nugent Z, Shaw S et al. Outcomes of patients with Crohn's disease improved from 1988 to 2008 and were associated with increased specialist care. Gastroenterology 2011; 141: $90-7$.

3. Loftus EV Jr, Schoenfeld P, Sandborn WJ. The epidemiology and natural history of Crohn's disease in population-based patient cohorts from North America: a systematic review. Aliment Pharmacol Ther 2002;16(1):51-60.

4. Smedh K, Andersson M, Johansson H, Hagberg T. Preoperative management is more important than choice of sutured or stapled anastomosis in Crohn's disease. Eur J Surg 2002; 168(3):154-157.

5. Donnellan CF, Yann LH, Lal S. Nutritional management of Crohn's disease. Ther Adv Gastroenterol, 2013; 6(3): 231-242.

6. Mäkelä JT, Kiviniemi H, Laitinen S. Risk factors for anastomotic leakage after left-sided colorectal resection with rectal anastomosis. Dis Colon Rectum 2003;46(5):653-660.

7. Suding P, Jensen E, AbramsonMA, Itani K, Wilson SE. Definitive risk factors for anastomotic leaks in elective open colorectal resection. Arch Surg 2008;143(9):907-911, discussion 911-912.

8. Van Gossum, A., Cabre, E., Hebuterne, X., Jeppesen, P., Krznaric, Z., Messing, B. et al. ESPEN guidelines on parenteral nutrition: gastroenterology. Clin Nutr 2009; 28: 415-427. 


\section{Pré e Pós operatório nas DII}

\section{A Visão do Cirurgião}

\section{André da Luz Moreira}

\section{Introdução}

A maioria dos pacientes com doença de Crohn (DC) necessitam de pelo menos um procedimento cirúrgico durante o curso natural da doença, frequentemente por estenoses ou fistulas. 0 risco cumulativo de ressecção intestinal na DC pode chegar a 70-80\% em 10 anos após o diagnostico. Na retocolite ulcerativa (RCUI), aproximadamente $30-40 \%$ dos pacientes são submetidos a cirurgia por doença refratária ao tratamento clínico, e em menor escala por displasia, neoplasia ou colite aguda grave.

Em grande parte das vezes, o tratamento clinico é levado ao extremo e a cirurgia somente é recomendada em ultimo caso. Consequentemente, no pré-operatório, os pacientes podem se apresentar desnutridos, imunossuprimidos e alguns com sinais de sepse intra-abdominal. Esses fatores estão associados a um risco aumentado de inúmeras complicações pós-operatórias e apresentam um grande desafio para o cirurgião. Portanto, a otimização pré-operatória na tentativa de diminuir esses riscos e trazer bons resultados cirúrgicos é imperativa na avaliação desses pacientes.

\section{Avaliação Nutricional}

A desnutrição pré-operatória aumenta as taxas de morbidade pós-operatória e o risco de sepse abdominal. Muitos pacientes são enviados ao cirurgião como último recurso e portanto chegam desnutridos por debilidade crônica. Entretanto, não existe um teste padrão para diagnosticar a desnutrição, uma vez que ela envolve uma avaliação com- 
plexa incluindo vários fatores. As deficiências nutricionais encontradas são resultado de uma ingesta inadequada de nutrientes devido a dor abdominal pós-prandial ou diarreia, má absorção devido as alterações da mucosa, a inflamação sistêmica que altera a síntese de proteínas e aumenta o catabolismo proteico e a efeitos colaterais de algumas medicações utilizadas no tratamento da doença inflamatória intestinal (DII). A complicação mais temida no pós-operatória é a deiscência de anastomose. Existem inúmeros estudos relacionando essa complicação com a hipoalbuminemia. Portanto, a otimização nutricional pré-operatória pode corrigir essa deficiência utilizando nutrição parenteral total (NPT) ou nutrição enteral (NE). A NPT administrada por pelo menos 20 a 30 dias antes da cirurgia pode restabelecer alguns parâmetros nutricionais e preparar o paciente metabolicamente para o trauma cirúrgico, reduzindo assim as complicações pós-operatórias. O suporte enteral (ex. dietas elementares ou oligomonoméricas) deve ser a via de preferência sempre que possível, por ser a via mais fisiológica, de menor custo, e com menos risco de sepse quando comparada a NPT.

\section{Manejo Farmacológico}

O principal tratamento clínico na DIl envolve a supressão da resposta imunológica utilizando corticoides, imunomoduladores e agentes biológicos. Entretanto, esses medicamentos podem estar associados a complicações pós-operatórias apesar de muitas evidências na literatura serem conflitantes.

Muitos pacientes com DII precisam de corticoides durante o curso da doença, 30-40\% desses pacientes com doença moderada ou grave ficam cortico-dependentes. Embora o cenário ideal fosse realizar uma cirurgia eletiva em pacientes que conseguiram descontinuar a corticoterapia por períodos prolongados, a realidade é que uma parcela significativa de pacientes chegam a cirurgia com doses variadas de corticoide devido a impossibilidade de suspender essa medicação a tempo. Durante o trauma cirúrgico, o organismo necessita de corticóides endógenos (resposta metabólica ao trauma) e essa resposta pode estar bloqueada pelo uso crônico de corticoides exógenos. Pacientes com insuficiência adrenal secundária à esse uso podem desenvolver hipotensão, taquicardia ou hipoglicemia no período pós-operatório uma vez que seu corpo não consegue compensar o estresse associado a 
cirurgia. Portanto, o uso de corticoides no perioperatório se justifica como uma medida preemptiva ou preventiva nos pacientes com risco de supressão do eixo Hipotalâmico-Hipofisário-Adrenal (HHA). Muito embora o grau de supressão possa estar associado tradicionalmente a dose e a duração da terapia, há poucos dados na literatura para apoiar essa teoria. Glicocorticóides equivalentes a $5 \mathrm{mg}$ de prednisona diários ou menos e administrados por menos de 3 semanas não parecem causar supressão clinica significativa do eixo HHA. Entretanto, os pacientes que receberam o equivalente a $15 \mathrm{mg}$ de prednisolona diário por mais de 3 semanas são suspeitos de ter essa supressão. Apesar de ser possível avaliar a supressão do eixo HHA através do teste da corticotropina, uma cobertura empírica para os pacientes de risco é uma abordagem mais prática na rotina dos cuidados peri-operatórios.

Os imunomoduladores também são frequentemente utilizados para manter remissão e poupar corticoide no tratamento clínico da DII. Assim como os corticoides, também há opiniões conflitantes em relação ao risco de complicações cirúrgicas em pacientes utilizando imunomoduladores, principalmente em relação a droga mais utilizada, a azatioprina. A recomendação atual sugere manter a azatioprina no período perioperatório, suspendendo no dia da cirurgia e reiniciando assim que o paciente esteja tolerando dieta oral.

Os agentes biológicos tem sido cada vez mais utilizados na DII e hoje em dia é comum a realização de cirurgias em pacientes recebendo anticorpos anti-TNF alfa. 0 uso desses agentes tem preocupado os cirurgiões em relação ao risco de complicações sépticas no pós-operatório. O risco de complicações em pacientes com DC ainda é um assunto controverso e o intervalo seguro ainda não foi determinado. Alguns autores recomendam a realização da cirurgia após 2 meses da ultima dose e o reinicio da terapia para profilaxia pós-operatória após 2 a 4 semanas da data da cirurgia. Na cirurgia para RCUI o uso de biológicos tem sido associado a um maior risco de complicações pós-operatórias, sugerindo uma maior utilização de três estágios na confecção de reservatórios íleo-anais.

\section{Manejo da Sepse}

A sepse abdominal na forma de fístulas e abscessos é relativamente comum na DC, ocorrendo em 10 a 30\% dos casos e represen- 
ta um fator de risco independente para complicações pós-operatórias. Tradicionalmente, os abscessos eram drenados cirurgicamente e a formação de estomas era frequente. Entretanto, atualmente, recomenda-se, sempre que possível, o uso de antibióticos parenterais associados ou não a drenagem percutânea guiada por imagem radiológica na tentativa de "esfriar" o processo infeccioso e evitar uma cirurgia na fase aguda, permitindo assim, uma intervenção cirúrgica eletiva com menos chance de realização de estomas.

\section{Profilaxia de Fenômenos Tromboembólicos}

Como em todas as cirurgias abdominais e pélvicas de grande porte, a profilaxia de complicações tromboembólicas deve ser realizada segundo os diretrizes sugeridas pelo the American College of Chest Physicians Evidence- Based Clinical Practice Guidelines (9th edition). 0 risco de trombose venosa profunda e embolia pulmonar está aumentado nos pacientes com DIl em até 3 vezes quando comparados com a população geral. Portanto, a profilaxia farmacológica pode não ser suficiente. Consequentemente, pacientes com DIl necessitando de cirurgia estão em alto risco para complicações tromboembólicas pós-operatórias e a profilaxia combinada, farmacológica e mecânica, deve ser considerada.

\section{Cuidados Pós-operatórios}

A profilaxia antibiótica deve ser suspensa nas primeiras 24 horas depois da cirurgia, ao menos que o paciente já tenha complicações sépticas abdominais prévias. Não há obrigatoriedade do uso de sonda nasogástrica após as cirurgias eletivas e a utilização de drenos abdominais deve ser restrito dependendo dos critérios de cada cirurgião. A dieta oral pode ser reiniciada precocemente principalmente nos casos em que a via laparoscópica foi utilizada associado a deambulação precoce e fisioterapia respiratória. A presença de enfermeiros especializados (estomaterapeutas) é importante nos pacientes submetidos a ostomias, auxiliando nos cuidados e na educação do paciente. Lembrando sempre, que o local do estoma deve sempre ser marcado no pré-operatório com o paciente acordado para melhor resultados funcionais e de qualidade de vida. 


\section{Referências bibliográficas}

1. Loftus EV Jr, Schoenfeld P, Sandborn WJ. The epidemiology and natural history of Crohn's disease in population-based patient cohorts from North America: a systematic review. Aliment Pharmacol Ther 2002;16(1):51-60

2. Yamamoto T, Allan RN, Keighley MRB. Risk factors for intraabdominal sepsis after surgery in Crohn's disease. Dis Colon Rectum 2000;43(8):1141-1145

3. White JV, Guenter P, Jensen G, Malone A, Schofield M; Academy MalnutritionWork Group A.S.P.E.N. Malnutrition Task Force A.S.P. E.N. Board of Directors. Consensus statement: Academy of Nutrition and Dietetics and American Society for Parenteral and Enteral Nutrition: characteristics recommended for the identification and documentation of adult malnutrition (undernutrition). JPEN J Parenter Enteral Nutr 2012;36(3):275-283

4. Schlaghecke R, Kornely E, Santen RT, Ridderskamp P. The effect of long-term glucocorticoid therapy on pituitary-adrenal responses to exogenous corticotropin-releasing hormone. N Engl J Med 1992;326(4):226-230

5. Bernstein CN, Blanchard JF, Houston DS, Wajda A. The incidence of deep venous thrombosis and pulmonary embolism among patients with inflammatory bowel disease: a population-based cohort study. Thromb Haemost 2001;85(3):430-434.

6. Zerbib P, Koriche D, Truant S, et al. Pre-operative management is associated with low rate of post-operative morbidity in penetrating Crohn's disease. Aliment Pharmacol Ther 2010;32(3):459465.

7. Colombel JF, Loftus EV Jr, Tremaine WJ, et al. Early postoperative complications are not increased in patients with Crohn's disease treated perioperatively with infliximab or immunosuppressive therapy. Am J Gastroenterol 2004;99(5):878-883 